\title{
Research progress on user experience metric
}

\author{
Wei Zhang1, a \\ ${ }^{1}$ School of Informatics and Computing, Indiana University Bloomington, Bloomington 47408, US \\ ànang446@indianaledu
}

Keywords: user experience, metric, usability, quantitative evaluation

\begin{abstract}
The Internet has converged the global information into the intangible network, expanding the amount of information while changing the traditional way of people access information, and has become an important way of people share, disseminate, and obtain information. At the same time, user experience has become a crucial criterion to measure Internet products, which are the tightly related products of users' daily life. User behaviors are influenced by living habits and customs, life attitudes, and values, which are the crucial elements need to be taken into consideration when analyzing the user experience. In this thesis, we will introduce the concept, components of user experience and measurements to quantify the user experience from performance metric, problem-based metric, subjective metric and physiological metric.
\end{abstract}

\section{Introduction}

American economists B. Joseph Pine II and James H. Gilmore claimed in their book Experience Economy that a new form of economy - the experience economy is being separated from the service economy since operators are pursuing unique selling points under the pressure of fierce market competition, which is the forth economic stage after product economy, commodity economy and service economy. The emergence of experience economy is the corollary of social productivity and social development, as well as the result of overall increasing of demand hierarchy. In the era of experience economy, as the representatives of psychological demand, spirit and emotions have surpassed the material demand and become the domain demand among all. Different scholars from various countries have proposed their own views and understandings on the concept of user experience.

Norman first came up with the term of "user experience", pointing out that a successful user experience has to meet the customers' needs with the prerequisite of not disturbing or annoying them. Secondly, it has to provide customers with succinct and elegant products so that the customers can have them with pleasure and delight. Additionally, it should be able to bring extra surprise to customers [1]. The meaning of user experience is constantly extending with the expansion of its content and structure. [2]

User experience is subjective, dynamic from time variations, environmental limited and different among individuals, which all demonstrate that there will never be identical user experiences. However, it is still possible to create similar user experience with certain designs when it comes to clear target customer groups, which requires in depth research and precise control to take all the circumstances of customers using the product into consideration.

In this thesis, by analyzing the concept and composition of user experience, we will try to figure out a set of standardized measurement methods in order to quantify the user experience.

\section{Definition of usability}

The development of usability research has replaced the concept of "'user-friendly" and it prompted the idea of "usability" in the field of human-computer interactions. Usability refers to guiding product 
designs and measuring product quality from the perspective of customers in order to obtain simplicity and pleasant while using the product.

The ISO 9241 International Standard outlines the definition of usability: The effectiveness, efficiency and satisfaction a product has while being used by a certain group of people in a specified context of use for a certain purpose. Effectiveness refers to the completeness and accuracy of user goal achievement. Efficiency is defined as the ratio between the accuracy and completeness of user goal achievement and the resources (such as time) used during the process. Satisfaction means the subjective thoughts of content and acceptance of users when using the product. [3] Jakob Nielsen proposed five attributes of usability, which are learnability, efficiency, memorability, less errors and satisfaction. [4] By synthesizing various definitions of usability, we will classify its concept into three layers in this thesis, which are:

Usable: Emphasizing that the product function is able to help users achieve their goals.

Easy to use: Refers to the efficiency and learnability of the interactions between users and the product while achieving goals.

Joy of use: Means the subjective feelings, such as satisfaction, of customers while using the product and after achieving the goals.

\section{Concept and compositions of user experience}

In recent years, the idea of user experience has caught greater attention after the concept of usability emerged, of which the theoretical research has also conducted a wide range of exploration. User experience refers to the things customers do, think, and feel while operating or using a product, or enjoying a service, including both objective values and subjective feelings offered by a product or service.

DA Norman classified emotional experience into three levels, which are visceral level, behavioral level, and reflective level. Visceral level indicates the stimulating feelings of the senses; behavioral level means the achievability and and satisfaction obtained while resolving the problem; reflective level refers to the deeper emotions and cognitions obtained after experiencing the other two levels. [6] The user experience towards a product contains of physical feelings, psychological feelings, cognitions and aesthetical feelings. In Moeslinger's opinion, user experience should consist of practical experience, sensory experience and emotional experience. [7] When it comes to the user experience of websites design, Garrett argues that the design should be based on strategy, scope, structure, skeleton and surface of the website. [8] (Shown in Figure 1)

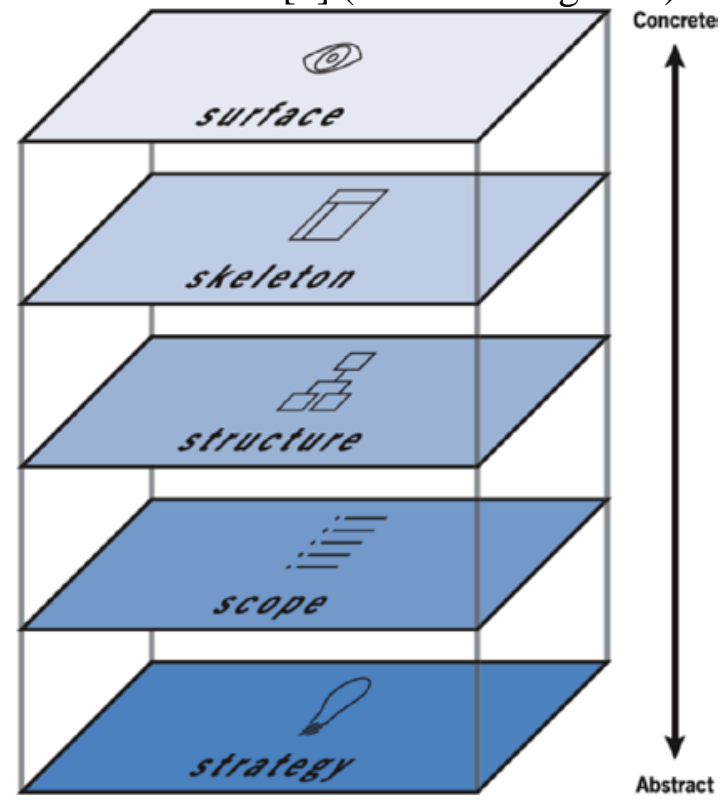

Fig. 1 The elements of user experience [8] 


\section{User experience metrics}

User experience is a subjective feeling of customers, which includes a wide range of content from different perspectives. The issue of how to quantify the user experience has become a new research subject. The idea of "measuring the user experience" was first proposed in 2004 when Robert Rubinoff brought up the four elements of user experience: branding, usability, functionality and content, based on which Robert conducted the quantitative analysis of user experience. In the book of Quantify the User Experience, Tom Tullis argues that usability focuses on the ability of achieving the goals with the use of a product, while user experience pays attention to the subjective feelings brought by the interactions, along with its consequences, between customers and products. [9] Tom Tullis extended the traditional meaning of usability measurement and quantify the user experience with a macroscopical view of usability.

Usability measurement can be divided as user-participated usability test and heuristic assessment. The user-participated usability test invites users to use the design prototype or the product to complete the operational task and to evaluate the interface usability by observing, recording, and analyzing user behaviors and relevant data. As for heuristic assessment, also known as the expert evaluation, is conducted by the experts who play the role of ordinary users and propose potential problems users may encounter when using the product, where the evaluation will be based on a series of guidelines. Nelson argued in his book "Usability Engineering” that when inviting 5 users to engage in the assessment can figure out $70 \%$ of usability issues, and he also put forward the general process of usability test and other precautions. [4] (Shown in Figure 2)

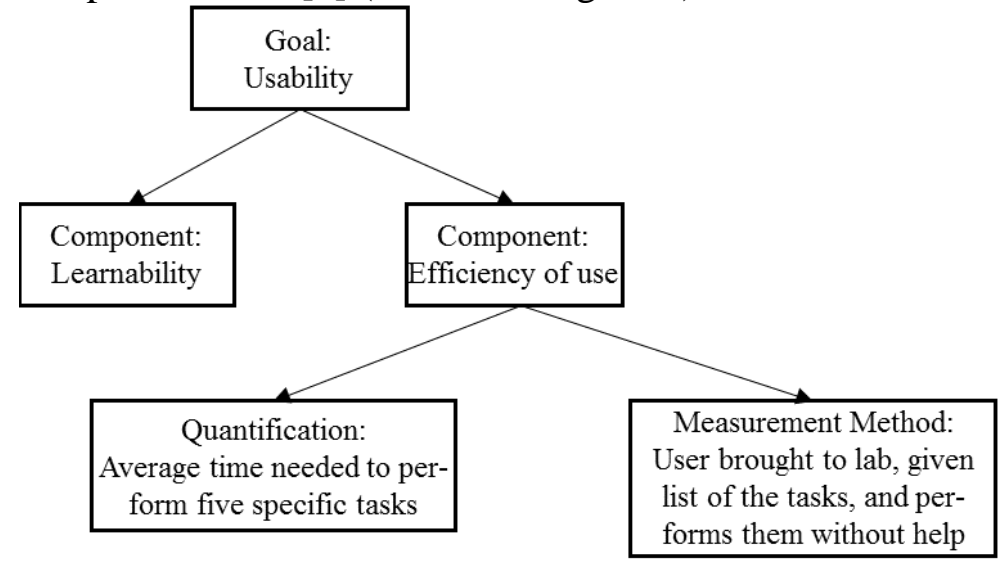

$$
\begin{gathered}
\text { Data-Collection Technique: } \\
\text { Stopwatch } \\
\text { (with rules for when to start } \\
\text { and stop the watch) }
\end{gathered}
$$

Fig. 2 The general process of Nielsen usability testing [4]

Performance metric. The purpose of network product development is to help users complete a specific goal, where a series of user behaviors will emerge along the way. These behaviors will form the basis of performance measurement indicators. Therefore, performance measurement is usually used in the user-participated usability test, which means giving users one or more than one tasks and certain targets to complete and recording the results of users using the network product during the process.

Since user behavior is objective and measurable, it is very valuable data for researching websites. Preece defines usability measurement as the systematic process for collecting data in order to get a better understanding of users and to know well of what it would be like for a certain group of users completing a certain task with the use of the product under specific conditions. There were a bunch of valuable user behavior data found in previous literatures. By measuring users' interest and satisfaction in web searching with implicit measurement, Fox et al. found out that elements such as 
the duration of stay on the web page, click through, what do users do after searching, how do users exit searching are crucial predictions of user satisfaction for search session. [10]

In accordance with the above analysis, we summarized several common measurement indicators:

Time of task: the time used of users to complete a particular task with the use of the product.

Successful ratio: the proportion of all the test users who complete the task, as well as the rate of success of each person in completing several tasks.

Errors: false steps took in the process of assessing and wrong operations, etc.

Efficiency: the relationship between the efforts put and the time used to complete the task, where the efforts are usually indicated by the number of operational steps, the number of pages viewed, and the amount of links clicked.

There are also rate of lost, rate of repeated page views and other indicators for different product task assessment with different purposes.

Problem-based metric. Network products are rich in content and complex in structure, hence a paragraph of semantic unclearness, a failure of a button or a structural unclearness are usability issues need to be improved. Users can provide priceless ideas to product design and product amelioration by discovering usability issues while using the product. Except for finding issues during usability assessment, a lot of network products also collect feedbacks from users after launching their product online by setting up the feedback windows for users in order to improve the products accordingly. This kind of problem discovering method is usually seen as a qualitative research approach. In order to prompt the amelioration of usability, the usability issues can be analyzed by quantitative approaches so that people can sort out the priority and arrange the order of improvement. Calculating the frequency of problems occurring and figuring out the severity of the problem are common ways used.

Subjective metric. Except for the objective methods mentioned above, the subjective feelings of users are crucial since user experience values the subjective feelings. By the approaches of questionnaire and direct contact with users, people can get the subjective feelings of the product design in order to predict the usability issues. Tullis defines the subjective metric as a measurement of self-reporting since the subjective data come from the user's inquiry. [9] Subjective metric can be divided into open questions and scale assessment these two categories.

Physiological metric. Physiological metrics are assisted by the equipment, which help user experience research by capturing the real response of human body. Main methods are eye tracking, facial recognition, skin conductance, and heart rate measurement. Emotions play a crucial role in the subjective experience of users, which has three compositions: intrinsic experience, behavioral performance and physiological awakening. [12] Where physiological awakening can be measured with the help of biological equipment. Common physiological indicators are EEG, heart rate, skin conductance, border electricity and so on. Emotion is a kind of subjective experience which is hard to be expressed through language. The measurement of the instrument can objectively reflect the user's emotional reaction and assist the user experience metric.

\section{Conclusion}

With the arrival of information era, a large amount of new diverse information emerged along the way, so does people's desire for higher level of information services. In order to obtain the acceptance of the service, service providers have to guarantee the outstanding user experience. Therefore, the quality of user experience has not only become one of the important research topics in academia, but also one of the key evaluation indexes of industrial community. This thesis summarizes the concept of user experience and measurement methods, and concludes that the user experience can be measured and quantified. This paper proves that the user experience can not only be quantified from the four aspects of task performance measurement, problem-based measurement, subjective measurement and physiological measurement, and the result of quantitative user experience evaluation is more helpful to optimize the product experience. It also provides the quantifying method of user experience based on the usability test. 


\section{Reference}

[1] Norman D, Miller J, Henderson. What you see, some of what's in the future, and how we go about doing it: $\mathrm{HI}$ at Apple Computer [C]. Conference companion on human factors in computing systems, Massachusetts, ACM, 1994: 155-155.

[2] Seapin D L, Senach B, Trousse B, et a1. User Experience: Buzzword or New Paradigm [C]. ACHI 2012, The Fifth International Conference on Advances in Computer-Human Interactions, Valencia, Netherlands, XPS, 2012: 336-341.

[3] JH Zhao. Design Psychology [M]. Beijing: Beijing Institute of Technology Press, 2004, 1.

[4] J Nielsen. Usability Engineering [M]. Beijing: Machinery Industry Press, 2004, 9.

[5] L Daniels. Understanding user experience [J]. Web Techniques, 2000, 5 (8): 42-43.

[6] DA Norman. Emotional design [M]. Beijing: Publishing House of Electronics Industry, 2005, 5-78.

[7] S Moeslinger. Technology at home: a digital personal scale [C]. Chi 97 Extended Abstracts on Human Factors in Computing Systems, 1997: 216-217.

[8] JJ Garrett. The elements of user experience [M]. New Riders, 2003. 